\title{
The double-dust chemistry phenomenon in PNe with [WC]-type central stars
}

\author{
J. V. Perea-Calderón ${ }^{1}$, D. A. García-Hernández ${ }^{2}$, \\ M. Bobrowsky ${ }^{3}$ and Pedro García-Lario ${ }^{2,4}$ \\ ${ }^{1}$ European Space Astronomy Centre, Madrid, Spain \\ email: jose.perea@sciops.esa.int \\ ${ }^{2}$ ISO Data Centre. European Space Astronomy Centre, ESA, Madrid, Spain \\ ${ }^{3}$ Computer Science Corporation/Space Telescope Science Institute. Baltimore MD, USA \\ ${ }^{4}$ Herschel Science Centre. European Space Astronomy Centre, Research and Scientific Support \\ Deparment of ESA, Madrid, Spain
}

\begin{abstract}
We present preliminary conclusions derived from the analysis of a sample of galactic PNe with [WC]-type central stars (WCPNe) observed with Spitzer/IRS. We report the detection of double-dust (C-rich and O-rich) chemistry in all stars in the sample for which a good S/N spectrum was obtained. Our observations reveal that the simultaneous presence of oxygen and carbon-rich dust features in the infrared spectra of WCPNe is not restricted to late/cool [WC]type stars, as previously suggested in the literature, but it is found to be a common feature associated to all WCPNe. In particular, mixed chemistry is observed in at least seven early/hot WCPNe in the sample. Various scenarios are proposed to interpret the results obtained in the framework of the chemical evolution of PNe.
\end{abstract}

Keywords. stars: abundances, AGB and post-AGB, evolution

\section{Introduction and Results}

Planetary Nebulae (PNe) are the result of the evolution of low and intermediate mass stars $\left(1-8 \mathrm{M}_{\odot}\right)$, where the circumstellar gas previously expelled during the Asymptotic Giant Branch (AGB) phase is ionized by the central star. AGB stars are characterized by increasing mass loss rates $\left(10^{-7}\right.$ to $\left.10^{-4} \mathrm{M}_{\odot} \mathrm{yr}^{-1}\right)$ which results in the development of circumstellar envelopes which emit strongly in the infrared and, thus, are easily detectable by Spitzer. The infrared emission from these circumstellar envelopes can still be very strong during the evolution of the star as a PN. An interesting class of PN are those with Wolf-Rayet ([WC]-type) central stars. Recent observations made with ISO SWS suggest that the envelopes of some of these WCPNe show a characteristic double-dust chemistry (Waters et al. 1998a,b; Cohen et al. 1999, 2002) which is not yet well understood. In particular, this phenomenon has been systematically observed so far only in late-type/cool WCPNe.

Here we report on the preliminary results from a systematic analysis made with Spitzer/IRS on a large sample of Galactic Bulge PNe. One of the most interesting conclusions derived from our observations is the detection of double-dust chemistry in all the WCPNe observed, irrespective of whether they are early-type/hot WCPNe or latetype/cool WCPNe. In Figure 1 we show four illustrative examples of the spectra obtained. The total sample comprises 19 WCPNe. All of them show the well-known family of infrared bands at $\lambda<15 \mu \mathrm{m}$ attributed to PAHs (C-rich) together with bands beyond $20 \mu \mathrm{m}$ identified as crystalline silicates (O-rich). 

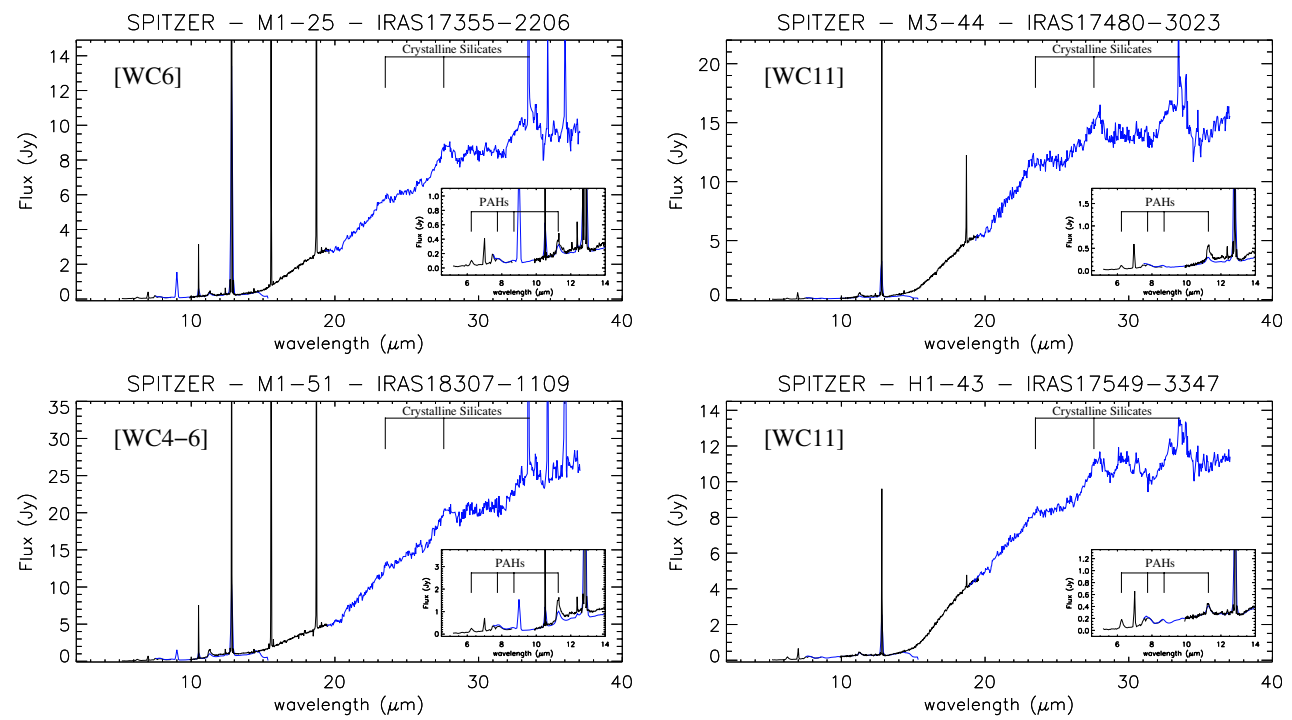

Figure 1. Representative Spitzer/IRS spectra of four WCPNe in our sample (early-type on the left side; late type on the right side). All of them show strong infrared continuum emission produced by thermal emission from the dust in the envelope. The simultaneous detection of PAH emission features in the 6-15 $\mu \mathrm{m}$ range (C-rich) together with prominent crystalline silicate emission features beyond $20 \mu \mathrm{m}$ (O-rich) reveal the dual-dust chemistry of these stars.

\section{Discussion}

The origin of the double dust chemistry phenomenon is unclear at present. Several mechanisms have been proposed to explain the observations:

i) Late thermal pulse at the very end of the AGB phase or shortly thereafter: a sudden switch in the observed chemistry can be produced as a consequence of a final dredge-up of C-rich processed material to the surface (Waters et al. 1998a). As a consequence of the enhanced mass loss, high temperature crystallization of the dust in the circumstellar shell could be triggered in parallel, affecting both the pre-existing O-rich material and the newly released $\mathrm{C}$-rich material.

ii) Hot bottom burning (HBB) termination at the end of the AGB phase: mass-loss reducing the envelope mass can de-activate the HBB but not the efficient dredge-up of C-rich material to the surface during the last few thermal pulses (Frost et al.. 1998).

iii) Binary star evolution: it has been suggested (Molster et al. 1999) that low temperature crystallization may take place in circumbinary disks. In this scenario, PAH emission is expected to be predominantly present in the scattering lobes (Waters et al. 1998b). The problem clearly deserves further analysis and needs to take into account the new observational constraints here provided.

\section{References}

Cohen, M., Barlow, M.J., Sylvester, R.J., Liu, X.-W., Cox, P., Lim, T., Schmitt, B., \& Speck, A.K. 1999, ApJL 513, 135

Cohen, M., Barlow, M.J., Liu, X.-W., \& Jones, A.F. 2002, MNRAS 332, 879

Frost, C.A., Cannon, R.C., Lattanzio, J.C., Wood, P.R., \& Forestini, M. 1998, A\& A, 332, L17

Molster, F.J., Yamamura, I., Waters, L.B.F.M., et al.. 1999, Nature 401, 563

Waters, L.B.F.M., Beintema, D.A., Zijlstra, A.A., et al.. 1998a, A\&GA 331, L61

Waters, L.B.F.M., Cami, J., de Jong, T., et al.. Nature 391, 868 